Derecho y Sociedad Revista del programa de Derecho Vol. 1 MMXVI, $\mathrm{N}^{\circ} 1$

Javier Isaac Ruiz Mestra ${ }^{1}$ p. $72-87$

javierisaac@gmail.com

Alba Lorena Torres Polo ${ }^{2}$

loren_torresp@hotmail.com

7201004

Sección 1

Avance de investigación versión final

Universidad de Córdoba

Junio 17 - 2016

\title{
EFECTOS DE LA SENTENCIA T-194 DE 1999 EN LA SOCIEDAD QUE HABITA LA CUENCA MEDIA Y BAJA DEL RIO SINÚ
}

\section{RESUMEN}

Este artículo - producto de un trabajo investigativo a nivel formativo-, realiza un análisis sociojurídico a la Sentencia T-194/99 con el fin de exponer sus efectos directos e indirectos. En el primer apartado se reflexiona sobre los antecedentes de este pronunciamiento judicial. En el segundo, se establece el procedimiento metodológico utilizado en el citado análisis sociojurídico. En el tercer apartado, se muestra los resultados obtenidos. El cuarto, presenta el punto de vista de ASPROCIG, que es la organización más relevante en este asunto, por ser la que logró incoar el proceso de solicitud de amparo de sus asociados contra las actuaciones de la Corporación Eléctrica de la Costa Atlántica y la Empresa Multipropósito Urrá S.A. El artículo concluye que la Universidad de Córdoba, inicie a través de su Facultad de Ciencias Económicas, Jurídicas y Administrativas estudios de fondo, que coadyuven a la solución de tales problemáticas. La tesis a demostrar es que el pronunciamiento de la Corte Constitucional por medio de la Sentencia T-194/99 es ejemplar, pero no acatado.

Palabras Claves: Humedales. Comunidad. INCODER. CVS. Debido proceso. Derechos fundamentales.

\footnotetext{
${ }^{1}$ I.A. M.Sc Ciencias Agronómicas. Estudiante del programa de Derecho. Universidad de Córdoba

${ }^{2}$ Licenciada en Educación Básica con énfasis en Ingles. Estudiante del programa de Derecho Universidad de Córdoba
} 


\section{Abstract}

This article - product investigative work to formativo- level, takes a socio-legal analysis to Judgment T-194/99 in order to expose their direct and indirect effects. In the first section, we examine the history of this judicial pronouncement. In the second, the methodological procedure used in that socio-legal analysis is established. In the third section, the results shown. The fourth presents the point of view of ASPROCIG, which is the most important organization in this case, by which managed to initiate the application process for defense of its members against the actions of the Electricity Corporation of the Atlantic Coast and the Company multipurpose Urrá SA The article concludes that the University of Cordoba, start through its Faculty of Economics, Legal and Administrative studies, to contribute to the solution of such problems. The thesis is to demonstrate that the decision of the Constitutional Court through Judgment T-194/99 is exemplary, but not abided.

Keywords: Wetlands. Community. INCODER. CVS. Correctly process. Fundamental Rights.

\section{PRESENTACIÓN}

En cumplimiento del objetivo fundamental del programa de Derecho de la Universidad de Córdoba, consistente en formar profesionales íntegros, con aptitudes interdisciplinarias que los distingan como ciudadanos éticos, con sólidos preceptos morales y competentes para desarrollar idóneamente el ejercicio del derecho, con conocimientos sociojurídicos, que les permita ocupar un lugar destacado en la comunidad por sus aportes en el Derecho Ambiental, y Derechos Humanos, el curso de Sociología Jurídica, -valiéndose de objetivos no declarados del derecho- inicia la tarea de realizar indagaciones en torno al déficit de la eficacia jurídica generada a partir del pronunciamiento de la Corte Constitucional en Sentencia T-194/99.

La intención es identificar y analizar los efectos directos e indirectos -esto es res indicata inter partes- producidos a partir del fallo proferido en la Sentencia T-194/99.

Para hacer el análisis de cómo el citado fallo ha generado efectos en la sociedad, se procedió a entrevistar a representantes de las partes involucradas en el proceso, además, se aplicó encuestas a una muestra de los habitantes de Lorica y de los alrededores de la Ciénega Grande de Lorica. 
Los efectos directos, se relacionan con las conductas ordenadas por el fallo, es decir, con los derechos creados a favor de las comunidades del bajo Sinú por la Corte Constitucional a partir de la adopción de medidas de armonización orientadas a "tutelar los derechos de los afiliados a la Asociación de Productores para el Desarrollo Comunitario de la Ciénaga Grande de Lorica-ASPROCIG-, a la participación y a un medio ambiente sano" (Expediente T-175.217).

\section{ANTECEDENTES}

En sus consideraciones, la Corte Constitucional sostiene que "hasta finales del siglo XVII, salvo por las obras de la ingeniería Zenú, el valle del Sinú conservó la flora y la fauna propias de los pantanos y delta costeros prácticamente inalterados" (Expediente T-175.217), “el pueblo Zenú entendía que los pantanos y las ciénagas tienen una estrecha relación con la dinámica fluvial e inundaciones periódicas, así que llevaron a cabo la construcción de obras hidráulicas que actuaban como verdaderos sistemas de amortiguación y almacenamiento de los excedentes caudales y lluvias locales (ASPROCIG, p. 24). Expresa la corte [...] "Pero durante los tres siglos siguientes, se tumbó el bosque bajo y se reemplazó con pasto para la ganadería extensiva, se desecaron progresivamente los pantanos, pozos, ciénagas y caños, se multiplicaron los asentamientos humanos a la ribera del río, las quebradas y los caños, se introdujo la agricultura intensiva $\mathrm{y}$, finalmente, los cultivos tecnificados con el uso de maquinaria, pesticidas y abonos industriales, así como algunas facilidades turísticas, de manera tal que, salvo por algunos manglares costeros, se puede afirmar que el valle quedó completamente deforestado y prácticamente desecado en la década de los sesenta y posteriores del siglo pasado y presente" (Expediente T-175.217)

Andrés Abelino Núñez Morales en representación de ASPROCIG, instauró acción de tutela el día 03 de julio de 1998 ante la Sala Penal del Distrito Superior de Montería, contra la empresa multipropósito URRA y solicitó se le ampararan los derechos a la vida, la salud, el trabajo y un medio ambiente sano.

El Tribunal Superior del Distrito Judicial de Montería, en fallo de primera instancia decidió negar la solicitud de tutela por improcedente el amparo de los derechos reclamados, 
manifestando que "los miembros de APROSIC cuentan con otros mecanismos judiciales para reclamar la indemnización de los perjuicios que afirman haber sufrido" (Expediente T175.217). Decisión que fue impugnada ante la Sala de Casación Penal de la Corte Suprema de Justicia, la cual confirmó la decisión impugnada el día 08 de septiembre de 1998 y argumentó que los miembros de la citada asociación tienen constitucional y legalmente vías expeditas como la acción de nulidad y restablecimiento del derecho. La Corte Constitucional revisa la presente acción y los fallos proferidos en trámite de las distintas instancias.

El problema examinado por la corte constitucional consistió en: "lo que resulta más preocupante para esta Corporación, es que el cambio de la Constitución Nacional por la Carta Política de 1991 no se reflejó en la actividad que cumplen las autoridades de los catorce municipios de la hoya hidrográfica y las del Departamento de Córdoba, para quienes parece no existir el deber social del Estado (C.P. art. 2), consagrado como principio fundamental y obligación de éste y de los particulares en el artículo 8 Superior, de proteger las riquezas naturales de la nación" (Expediente T-175.217), y prosigue:

1. Proceso de degradación medioambiental que afecta la cuenca del rio Sinú

2. Desecación de los cuerpos de agua, contaminación de los sistemas de humedales del Sinú y desempeño de entes públicos

3. Patrón de poblamiento, desarrollo y costos asociados a las inundaciones periódicas de la cuenca hidrográfica del rio Sinú

4. Prohibición de adjudicar como baldíos terrenos públicos de las ciénagas de Córdoba y áreas de la hoja del Sinú por parte del INCORA

5. Explotación del recurso íctico, las prácticas dañinas y el control ineficiente de la cuenca del Sinú

6. Impacto en el recurso ictiológico

7. Proceso de consulta y concertación por el impacto medioambiental

Decisión que fue impugnada y en segunda instancia "la Sala de Casación Penal de la Corte Suprema de Justicia confirmó la decisión impugnada (8 de septiembre de 1998), y anotó que los disensos contenidos en la demanda de tutela aquí presentada tienen, constitucional y legalmente, cabida en las acciones de nulidad y restablecimiento del derecho, 
e incluso en la suspensión provisional, de conformidad con los artículos 84, 85 y 152 y siguientes del Código Contencioso Administrativo, acciones éstas referidas a la licencia ambiental que el INDERENA otorgó mediante resolución 0243 de abril 13 de 1993 a la Corporación Eléctrica de la Costa Atlántica -CORELCA-, para la etapa de construcción del Proyecto Hidroeléctrico Multipropósito de Urrá I" (folio 9 del primer cuaderno)" (Ibíd, p. 4), negando los derechos de los demandantes; la Corte Constitucional revisó en control de constitucionalidad la tutela en comento y en fallo T-194 de 1999 resolvió:

Primero. "REVOCAR parcialmente los fallos proferidos por la Sala de Casación Penal de la Corte Suprema de Justicia el 8 de septiembre de 1998 y la Sala Penal del Tribunal Superior del Distrito Judicial de Montería el 3 de julio de 1998 y, en su lugar, tutelar los derechos de los afiliados a la Asociación de Productores para el Desarrollo Comunitario de la Ciénaga Grande de Lorica-ASPROCIG-, a la participación y a un medio ambiente sano; y confirmar la sentencia de segunda instancia, en cuanto negó el amparo de los derechos a la vida, la salud y el trabajo" (Expediente T-175.217, p. 14)

Segundo. "ORDENAR a los Personeros, Alcaldes y Concejales de Tierralta, Valencia, Montería, Cereté, Lorica, San Bernardo del Viento, Purísima, Chimá, San Pelayo, Ciénaga de Oro, San Carlos, Momil, San Antero y Moñitos, que procedan de inmediato a: 1) suspender toda obra de relleno y desecación de pantanos, lagunas, charcas, ciénagas y humedales en el territorio de esos municipios, salvedad hecha de las que sean indispensables para el saneamiento; 2) adelantar las actuaciones administrativas de su competencia e instaurar las acciones procedentes para recuperar el dominio público sobre las áreas de terreno de los cuerpos de agua que fueron desecados y apropiados por particulares; 3) regular la manera en que se hará exigible en esos municipios cumplir con la función ecológica que le es inherente a la propiedad (C.P. art. 58), establecer y cobrar las obligaciones que de tal función se desprendan para los particulares y entes públicos; y 4) revisar los planes y programas de desarrollo económico y social, para dar prioridad a las necesidades que se derivan de : a) el tratamiento y vertimiento de las aguas negras, b) la recolección y disposición de basuras, y c) la recuperación de los cuerpos de agua. Se ordenará también a la Gobernación del Departamento de Córdoba que proceda de igual forma, y coordine el cumplimiento de 
tales tareas por parte de los municipios mencionados, sometiéndose a las políticas del Ministerio del Medio Ambiente sobre la materia. El Gobernador informará sobre la manera en que se acaten estas órdenes al Tribunal Superior de Montería -juez de tutela en primera instancia-, a la Procuraduría y a la Contraloría Departamentales, a fin de que éstas ejerzan los controles debidos" (Ibíd, p. 15)

Tercero. "ORDENAR al Instituto Colombiano para la Reforma Agraria -INCORA-, que suspenda inmediatamente la política irregular de adjudicar como baldíos los terrenos públicos ubicados en las márgenes de las ciénagas de Córdoba, y las áreas que resulten del relleno de los humedales, lagunas, pozos, lagos o caños de la hoya del Sinú” (Ibíd, p. 15)

Cuarto. “ORDENAR a la Corporación Autónoma Regional de los Valles del Río Sinú y del San Jorge -CVS- y al Ministerio del Medio Ambiente que, en el marco de su participación en la prevención y mitigación del impacto de la hidroeléctrica, incluyan los estudios de factibilidad de un subprograma centrado en la formación de los pescadores para que éstos asuman las responsabilidades que les corresponden en la protección de la diversidad e integridad del ambiente y la conservación ecológica del medio en el que habitan y laboran, y para que puedan mejorar su nivel de vida con actividades de doble propósito como los zoocriaderos de iguanas e hicoteas" (Ibíd, p. 15).

Quinto. “ORDENAR al Ministerio del Medio Ambiente, la Gobernación de Córdoba y la CVS que dentro de las cuarenta y ocho (48) horas siguientes a la notificación de este fallo, den cumplimiento al acuerdo por medio del cual esas entidades se comprometieron a ejecutar programas de limpieza de caños, empleando a los pescadores demandantes” (Ibíd, p. 15).

Sexto. "ORDENAR que el Ministerio del Medio Ambiente, el Ministerio de Minas y Energía, la Gobernación de Córdoba, la Empresa Multipropósito Urrá S.A., y los entes territoriales que recibirán regalías por la operación de la hidroeléctrica Urrá I, concurran a financiar la asesoría que requieran las comunidades afectadas con la obra en el ejercicio del derecho a la participación efectiva que les otorga la Constitución Política” (Ibíd, p. 16). 
Séptimo. “ADVERTIR al Ministerio del Medio Ambiente que, dentro del marco general de lo decidido en la sentencia T-652/98, deberá prestar especial atención al impacto previsible de las aguas del embalse Urrá I sobre las especies reofílicas de la cuenca, y sólo conceder la licencia para el llenado de la presa y funcionamiento de la hidroeléctrica, cuando se garantice la adopción de las medidas necesarias para que el aprovechamiento del agua en la producción de energía, no signifique la extinción del recurso ictiológico que aparece como gravemente amenazado" (Ibíd, p. 16).

Octavo. "COMUNICAR esta providencia al Tribunal Superior del Distrito Judicial de Montería para los efectos previstos en el artículo 36 del Decreto 2591 de 1991” (Ibíd, p.16).

\section{METODOLOGÍA}

El tema de los efectos de los fallos judiciales ha sido abordado por autores como Cesar Rodríguez, Michael McCann, Gerald Rosenberg, Michael Klarman, Malcolm Feeley, y Edward Rubin entre otros. El hilo de discusión seguido por estos teóricos es determinar los cambios surgidos en el derecho a partir de la intervención de los jueces, para lo cual fijan preguntas que orientan la discusión: “¿cómo evaluar el impacto de una decisión judicial? En términos más amplios, ¿cómo determinar los efectos de la judicialización de problemas sociales? ¿cómo medir el impacto de la transformación de una controversia política, económica o moral en un litigio?” (Rodríguez, 2011, p. 1679). La respuesta a este tipo de cuestionamientos habitualmente es ordenada en dos grupos: los que asumen el problema de los efectos directos desde una perspectiva neo-rrealista y los constructivistas.

En efecto, la perspectiva neo-rrealista aporta los criterios metodológicos idóneos para analizar las derivaciones surtidas por la Sentencia T-194/99 al amparar los derechos a los afiliados de ASPROCIG, por cuanto promueve la idea de que "los tribunales existen no sólo para enjuiciar a los delincuentes sino también para reafirmar derechos", tal y como se estableció en el caso Brown vs. Junta Escolar en 1954 emitido por el Tribunal Supremo de Estados Unidos. En este orden, -el neoconstitucionalismo- es la línea jurídica que garantiza la igual protección de los derechos. 
Esta perspectiva teórica es apoyada por Werner Kägi citado por Luís Prieto Sanchís al sostener que "la Constitución expresa jurídicamente los contenidos esenciales que integran a dicha comunidad, es decir los derechos fundamentales y la organización del poder político" (2003, p.24). Este planteamiento se soporta igualmente en Kelsen al expresar que "desde una perspectiva de la supremacía formal, la Constitución prevalece sobre la ley, esta sobre los reglamentos y así sucesivamente. Pese a este mandato, se pueden derivar dos consecuencias: una, que la Constitución es una lex legis - ley de leyes - en tanto se encuentra en el vértice de las demás normas, y prima en caso de conflicto sobre ellas; y norma normarun — norma fuente de normas - , en tanto constituye la norma matriz para la creación de las demás normas del sistema jurídico" (Ibíd, p. 24).

Sin embargo, Oscar Arismendy Martínez sostiene que a pesar de tener "una Constitución ecológica en nuestro país, la realidad parece no estar impactada por este constitucionalismo, lo cual se debe a la existencia de un derecho formalista que se valida apriorísticamente; [...] — precisa el autor- que existe un orden jurídico y un orden socialeconómico que no se integran al derecho en el momento de resolver un conflicto" (2009, p. 2) y amplía su consideración al citar a Evelio Daza y decir que "en el Estado social, el derecho se legitima por el contenido real, material de sus proposiciones y sus incidencias sociales, no por su validez formal" (Ibíd, p. 2) por lo tanto el profesor Arismendy explica que "el derecho revela su eficacia cuando es capaz de corregir conductas o hechos que ponen en peligro la convivencia social, cuando se supera la antinomia orden sociales vs orden jurídico. La dinámica misma del comercio, de la industria y en general de la actividad económica capitalista, puede convertirse en la causa de males tan graves o peores que los derivados de la violación de derechos subjetivos" (Ibid, p. 2).

Sumado a los alcances constitucionales, el presente trabajo de investigación formativa realizó entrevistas no estructuradas a miembros de la Asociación de Productores para el Desarrollo Comunitario de la Ciénaga Grande de Lorica (ASPROCIG) y a entidades como INCODER y la Corporación Autónoma Regional de los Valles del Río Sinú y del San Jorge (CVS). 


\section{RESULTADOS}

A continuación se muestran algunos de los resultados obtenidos en las indagaciones realizadas a los actores del problema -a los cuales se les aplicó encuestas tipo descriptiva-, con la intención de identificar y analizar sus percepciones en torno al tema de los efectos generados a partir del fallo de la Corte Constitucional según expediente T-175.217. Así mismo, se acopió fuente documental suministrada por los miembros de ASPROCIG a la cual se le realizó un análisis documental con el propósito de extraer los aspectos más relevantes. También se analizó información obtenida de medios de comunicación local relacionadas con el tema del citado fallo.

\section{Primeros Hallazgos}

\section{Apropiación indebida de tierras por desecación de la Ciénaga Grande de Lorica}

Para la Asociación de Productores para el Desarrollo Comunitario de la Ciénaga Grande de Lorica (ASPROCIG), la apropiación indebida de tierras por desecación de la Ciénaga Grande de Lorica, ha sido un proceso en el que los grandes propietarios de la región -utilizando su poder- se han aprovechado de la desecación de diferentes cuerpos de agua para su beneficio particular. Uno de los miembros de esta organización, entrevistado sostuvo que [...] "Hace muchos años esos humedales extensos estaban llenos de agua, no sabíamos cuál era la Ciénaga o cuál era el Río cuando venía la creciente. Cuando aparece la carretera, se divide la Ciénaga y en la margen izquierda viniendo de Montería quedaba el Río Sinú y a la derecha la Ciénaga, todo este espacio era un complejo cenagoso incluyendo la zona del delta, todo esto se la robaron, nosotros mismos hemos colaborado de alguna forma con eso, nosotros veníamos organizados, [...] pero empiezan los problemas de desalojo porque los señores grandes terratenientes de por acá son los que empiezan desecar para meterle sus productos el arroz, maíz. Luego aparece el gran proyecto de Córdoba y Colombia que desangra a los cordobeses URRA"3.

\footnotetext{
${ }^{3}$ Estos son aparte de testimonios tomados de entrevistas aplicadas a miembros de ASPROCIG, la cual fue transcrita literalmente de su narración original
} 
En la actualidad, los derechos de uso y control del territorio que los campesinos y pescadores poseen a través de diferentes formas de tenencia de la tierra: titulación, arriendo y ocupación de hecho o propiedad; compiten con los intereses de los grandes ganaderos de la región, creando con ello, una situación de conflicto que deteriora la condición socioeconómica -en especial- de campesinos y pescadores y el agotamiento de los recursos naturales que el medio les ofrece. El mismo entrevistado señala que [...] "el INCORA les permitió que muchos de ellos legalizaran parte de esas tierras", esta información -según la fuente-fue corroborada por el INCODER, proceso que de acuerdo a la fuente de ASPROCIG se dio en complicidad con las autoridades del departamento de Córdoba y la Oficina de Instrumentos Públicos de la ciudad de Lorica de la época, inclusive gente que hizo parte de las administraciones, dicen que tienen fincas en la Ciénaga, no lo podemos entender, ¿fincas en la ciénaga?, inclusive la alcaldesa encargada de la administración anterior tiene tierra en la ciénaga".

Lo que reflejan los resultados de las entrevistas, es que lo ordenado por la Corte Constitucional mediante Sentencia T_194/99 muy poco se ha cumplido. Miembros de ASPROCIG señalan que "adquirieron un predio cerca a la Ciénaga para desarrollar un proyecto de piscicultura en el marco de los acuerdos existentes con distintos organismos del Estado, para lo cual hemos hecho los tramites de solicitud de licencias deslinde del predio en acatamiento a toda la parte legal, sin embargo, funcionarios de la Alcaldía de Lorica manifestaron que no se podía deslindar de las escrituras esa parte que a nosotros nos corresponde como vecinos de la Ciénaga, fíjense ustedes!!!, para nosotros sí aplica, pero para ellos no, por eso la mayoría de los proyectos de ASPROCIG todavía no han sido realizado por esa razón".

"Ahora nos resulta otro problema con la realización de la nueva carretera que va a pasar por la Ciénaga, nos dicen que no hay que hacer consulta previa porque no hay comunidades negras, no hay indígenas, ni pescadores ni campesinos, la Ciénaga fue declarada distrito de manejo integral por la CVS o sea que parte de esa legislación la cambiaron para que la variante pueda pasar por la ciénaga" (Testimonio I, 04/05/2016 ). 
El no cumplimiento del fallo de la Sentencia T-194/99, se reafirma cuando en su entrevista, él mismo entrevistado señala que son las mismas autoridades quienes desconocen lo proferido por la Corte [...] "Lo paradójico del asunto es que las autoridades siguen "comprando" terrenos en ese sitio que son los que tienen que velar por el cuidado del ambiente, en contra de lo que dice la sentencia restitúyase al dominio público todos los humedales que fueron desecados, y no se ha hecho nada, ha sucedido lo contrario se han ido desecado más humedales, esto está generando una bomba de tiempo porque en cualquier momento las comunidades se van a reventar" (Testimonio II, 04/05/2016 )

[...] "La gobernación no ha hecho nada que nosotros conozcamos. La CVS en el Plan de Mitigación de Impactos, construyo 42 proyectos para piscicultura organizados en tres fases, se hizo la primera fase, la segunda y la tercera fase no ha sido posible que se realizase. La primera fase fue para pescadores que fueron impactadas en primera instancia que son la gente que más hacia uso del recurso de la Ciénaga, en segunda instancia organizaciones de campesinos que aunque no son pescadores pero también fueron afectados por el tema de Urrá a estos los cobijaba 7 proyectos, y en tercera instancia para personas que se ocupaban para expender las carnes del pescado, estos proyectos buscaban que se pudieran fortalecer todas las partes productivas del proyecto, pero sólo se ha realizado la primera fase" (Testimonio III, 04/05/2016 )

La misma fuente indica: [...] "No se ha recibido capacitación de la CVS ni de Urrá, nosotros como organización estamos metidos en el tema del cambio climático con el Comité Técnico al Plan de Adaptación, el cual estamos liderando, entre los cuales está la UPB, la Universidad de Córdoba, Urrá y Cerro Matoso" (Ibíd, 2016).

En relación a la información documental, la tabla 1 muestra la información suministrada por ASPROCIG relacionada con el monitoreo de prevención y mitigación de impacto que realiza la Corporación Autónoma Regional de los Valles del Río Sinú y del San Jorge -CVS- vinculado con la desecación de los cuerpos de agua en la Ciénaga Grande de Lorica generados por el funcionamiento de la Hidroeléctrica Urrá: 
Tabla $1^{4}$. Actividades de monitoreo realizado por la CVS

\begin{tabular}{|c|c|c|c|}
\hline $\begin{array}{l}\text { MONITOREO } \\
\text { REALIZADO }\end{array}$ & CUMPLIMIENTO & $\begin{array}{c}\text { SUSTENTACIÓN } \\
\text { TÉCNICA }\end{array}$ & PROPUESTA \\
\hline $\begin{array}{l}\text { Plan de Ordenamiento } \\
\text { de la cuenca }\end{array}$ & $0 \%$ acciones & $\begin{array}{l}\text { Uso indiscriminado de } \\
\text { suelos distintas a su } \\
\text { vocación natural }\end{array}$ & $\begin{array}{l}\text { Convocar a comunidades } \\
\text { y socializar y concertar } \\
\text { acciones sobre este } \\
\text { proyecto }\end{array}$ \\
\hline $\begin{array}{l}\text { Liberación de } r \text { tierra } \\
\text { para extender la } \\
\text { frontera } r \text { agrícola } \\
\text { intensiva y la ganadería } \\
\text { extensiva. }\end{array}$ & $\begin{array}{l}0 \% \text { acciones tendientes a } \\
\text { favorecer las poblaciones } \\
\text { que siempre han hecho } \\
\text { uso de la ciénaga. }\end{array}$ & $\begin{array}{l}\text { Apropiación de los } \\
\text { terrenos de la ciénaga por } \\
\text { parte de terratenientes. }\end{array}$ & $\begin{array}{l}\text { Dar el estado concesión } \\
\text { de estos terrenos en un } \\
\text { porcentaje a los } \\
\text { campesinos y el resto } \\
\text { como patrimonio del } \\
\text { mismo. }\end{array}$ \\
\hline Deslinde de humedales & $\begin{array}{l}0 \% \text { en toma de acciones } \\
\text { y cumplimiento } \\
\text { sentenciar corte } \\
\text { constitucional }\end{array}$ & $\begin{array}{l}\text { Sentencia T } 194 / 99 . \\
\text { Acta de reunión del } 13 \text { de } \\
\text { abril/99. Ayuda de } \\
\text { memoria agosto } 2 \text { y } 3 / 99, \\
\text { pág. } 6 \text { y } 123 \text {. Párrafos } 1, \\
2 \text { y } 3\end{array}$ & $\begin{array}{l}\text { Deslinde de la Ciénaga } \\
\text { Grande, Miramar, } \\
\text { Corralito, La Pacha, } \\
\text { Baño, Marcella, Vichal y } \\
\text { el Tabique. } \\
\text { Cumplimiento inmediato }\end{array}$ \\
\hline $\begin{array}{l}\text { Simulación caudal río } \\
\text { Sinú }\end{array}$ & $\begin{array}{l}0 \% \text { en toma de acciones } \\
\text { para evitar este proceso }\end{array}$ & $\begin{array}{l}\text { Desestabilización de } \\
\text { taludes, desecamiento de } \\
\text { Ciénagas, Salinización } \\
\text { de Suelos y otros }\end{array}$ & $\begin{array}{l}\text { Cumplimiento de la } \\
\text { licencia } 0838 \text { de octubre } \\
5 \text { de } 1999 \text { en cuanto a la } \\
\text { simulación de caudales } \\
\text { para mayor eficiencia del } \\
\text { mismo. }\end{array}$ \\
\hline $\begin{array}{l}\text { Rehabilitación } \\
\text { Bugre }\end{array}$ & $\begin{array}{l}0 \% \text { acciones que } \\
\text { permitan el rescate del } \\
\text { Caño }\end{array}$ & $\begin{array}{lr}\begin{array}{lr}\text { Disminución de los } \\
\text { caudales del } \\
\text { sedimentación }\end{array} \\
\begin{array}{l}\text { contaminación } \\
\text { mismo. }\end{array} & \text { del } \\
& \\
\end{array}$ & Dragado total del caño \\
\hline
\end{tabular}

Fuente: Archivo ASPROCIG

La tabla 1, evidencia el incumplimiento del fallo de Corte a través de la Sentencia T194 de 1999, se evidencia que han sido nulas las acciones implementadas por los entes de control para la inspección y vigilancia.

De la misma manera, el Meridiano de Córdoba en nota periodística del 9 de febrero de 2014, ubicado en la Sección Bajo Sinú, señala que "de las 38 mil 840 hectáreas de tierra que tenía la Ciénaga Grande solo quedan 23 mil en humedal. El resto ha sido desecado por la misma gente con la construcción de terraplenes en medio de la Ciénaga para cultivos y potreros para pastar ganado" (Equipo de redacción, 2014).

Estos resultados se encuentran en estrecha relación con lo planteado en el Informe realizado por la CVS y Universidad Nacional (2005) que señala que, "la estructura de tenencia de la tierra en el complejo lagunar del Bajo Sinú, al igual que en Colombia, en la

\footnotetext{
${ }^{4}$ La información de la tabla 1, es una adaptación del original en físico entregado por ASPROCIG.
} 
cuenca hidrográfica del río Sinú y en el departamento de Córdoba, se han enmarcado en un modelo de latifundios destinados en la mayoría de los casos a la ganadería extensiva, que continúa absorbiendo los minifundios, e introduciendo tierras pertenecientes a la zona de anegación del complejo a través de procesos de adecuación de las mismas, mediante la construcción de canales de drenaje, sistemas de bombeo y diques" (2005, p, 21).

Sobre este tema, el periodista Carlos Pulgarin publicó el 20 de noviembre de 1998 una nota periodística en el diario el Tiempo en el que sostenía "que ante la amenaza a la que están sometidos los humedales, es vital proteger el ecosistema mediante la declaración de parque regional natural, lo que permitirá la planificación y el ordenamiento territorial de la zona, con participación institucional y comunitaria. El proyecto obligaba a su alinderamiento y conservación” (p, 1). "El Parque Natural de Carácter Regional de la Ciénaga Grande del Bajo Sinú estará conformado por las 38.843 hectáreas de zonas de humedales que hacen parte de la Ciénaga Grande de Lorica" (Ibíd, 1) La propuesta fue discutida por el Congreso de la Republica a través del proyecto de ley número 113 de 1998, fue archivado luego de primer debate por los miembros de la Comisión Quinta Constitucional Permanente de la Cámara de Representantes, presidida por el honorable Representante José María Imbett Bermúdez, el 2 de junio de 1999, con cinco (5) votos positivos y siete (7) votos negativos como consta en acta $\mathrm{N}^{\circ} 016$ de 1999 Cámara.

\section{CONCLUSIÓN}

Inicialmente hay que indicar que este trabajo de investigación formativa exigió el uso de diversos métodos para la recolección de la información, que incluyó la aplicación de diez (10) entrevistas semiestructuradas, con una duración promedio de una hora, con actores clave del caso.

Segundo, el trabajo de campo incluyó la observación participativa en diversos espacios de seguimiento de la Sentencia, sumado a la aplicación de encuestas a las comunidades beneficiadas por el pronunciamiento judicial.

Tercero, el estudio implicó un análisis sistemático de la documentación producida por la Corte y su posterior seguimiento del caso, así como los numerosos informes y publicaciones elaborados por las entidades públicas y por las organizaciones de la sociedad.

Cuarto, analizamos las cifras y los indicadores producidos por los diferentes actores del caso para medir la evolución de la situación de la población afectada por las disposiciones de la Sentencia T-194 de 1999 y los autos posteriores.

Finalmente, dado nuestro interés en examinar los efectos simbólicos e indirectos a la par con los instrumentales y directos, es importante decir que este trabajo, no consultó de 
manera directa a los organismos, encargados por la Sentencia, de ser quienes debían ejercer la vigilancia y control sobre el fallo proferido por la Alta Corporación, asunto que se constituye en otro tema de investigación. Pero, el estudio preliminar incluyó una revisión de las notas de prensa sobre los impactos ambientales en la Ciénega Grande de Lorica publicadas en medios escritos de mayor difusión nacional y regional (el diario El Tiempo y El Meridiano de Córdoba).

Con respecto a los resultados preliminares de nuestra investigación formativa podemos concluir que, el fallo de la Corte Constitucional por medio de la Sentencia T-194/99 ha sido INCUMPLIDO, tanto por los Entes de control para la inspección y vigilancia, como por los Organismos Municipales y por las Empresas Estatales.

Se recomienda que profundizar este estudio, mediante la estructuración de un proyecto de investigación y articularlo a la Línea de Derecho Ambiental adscrita al programa de Derecho de la Facultad de Ciencias Económicas, Jurídicas y Administrativas de la Universidad de Córdoba. 


\section{Bibliografía}

Arizmendy Martínez, Oscar. (2009). Protección o desprotección del Derecho al Medio Ambiente: Un análisis desde la Teoría Crítica. Disponible en: En:http://www.monografias.com/trabajos71/proteccion-derecho-medioambiente/proteccion-derecho-medio-ambiente2.shtml.

Asociación de Productores de la Ciénaga Grande, ASPROCIG. (2006). Cultura y territorio. Diez años de trabajo en la cuenca baja del río Sinú. El Molde Gráficas Ltda. Bogotá

(2016) Archivo físico.

Análisis de la licencia Urrá. (Documento interno de trabajo). Fotocopiado

Beyond the Courtroom (2009) The Impact of Judicial Activism on Socioeconomic Rights in Latin America. Disponible en http://www.corteidh.or.cr/tablas/r27171.pdf

Congreso de La Republica. (2001). Gaceta del Congreso $N^{\circ} 199$ En: http://www.imprenta.gov.co/gacetap/gaceta.mostrar_documento?p_tipo=69\&p_num ero $=016 \& p \_$consec $=3551$

CVS - Universidad Nacional de Colombia. (2005). Diagnóstico de la Dinámica Hídrica, Tenencia y Ocupación del Complejo Lagunar del Bajo Sinú: análisis de tenencia y ocupación de la tierra., Universidad nacional de Colombia. Informe final. Medellín.

Daza, Evelio. (2004). El saber en el Estado social de derecho. En: Filosofía del derecho y filosofía social. Memorias del tercer congreso nacional, Barranquilla.

Gil, R. (2009). El Neoconstitucionalismo y los derechos fundamentales. En: http://www.juridicas.unam.mx/publica/librev/rev/qdiuris/cont/12/cnt/cnt3.pdf

Prieto Sanchís, L. (2003). Justicia Constitucional y Derechos Fundamentales. Ed. Trotta. Madrid.

Pulgarin, C. (1998) A Salvar la Ciénaga de Lorica Nota de Prensa: El Tiempo, 20 de noviembre Disponible en: En: http://www.eltiempo.com/archivo/documento/MAM851832

Rodríguez, C. (2011). Evaluando el impacto y promoviendo la implementación de las sentencias estructurales sobre DESC en Colombia. En: https://docs.escrnet.org/usr_doc/Rodriguez-Colombia-espa\%C3\%B1ol.pdf

Equipo de redacción (9 de febrero de 2014). La Ciénaga Grande del Bajo Sinú se seca. El Meridiano de Córdoba Recuperado de: 
http://elmeridianodecordoba.com.co/region/item/53098-la-ci\%C3\%A9naga-grandedel-bajo-sin\%C3\%BA-se-seca

Como citar este artículo

AVANCE DE INVESTIGACIÓN Ruiz Mestra J.; Torres Polo L. (2016) Efectos de la Sentencia T-194 de 1999 en la Sociedad que habita la cuenca Media y Baja del Rio Sinú. Derecho y Sociedad, 1(1), 47-60. Universidad de Córdoba. Montería. 